\title{
125-GHz Microwave Signal Generation Employing an Integrated Pulse Shaper
}

\author{
Liao, Shasha; Ding, Yunhong; Dong, Jianji; Wang, Xu Dong; Zhang, Xinliang
}

Published in:

Journal of Lightwave Technology

Link to article, DOI:

10.1109/JLT.2017.2698598

Publication date:

2017

Document Version

Peer reviewed version

Link back to DTU Orbit

Citation (APA):

Liao, S., Ding, Y., Dong, J., Wang, X. D., \& Zhang, X. (2017). 125-GHz Microwave Signal Generation Employing an Integrated Pulse Shaper. Journal of Lightwave Technology, 35(13), 2741-2745.

https://doi.org/10.1109/JLT.2017.2698598

\section{General rights}

Copyright and moral rights for the publications made accessible in the public portal are retained by the authors and/or other copyright owners and it is a condition of accessing publications that users recognise and abide by the legal requirements associated with these rights.

- Users may download and print one copy of any publication from the public portal for the purpose of private study or research.

- You may not further distribute the material or use it for any profit-making activity or commercial gain

- You may freely distribute the URL identifying the publication in the public portal

If you believe that this document breaches copyright please contact us providing details, and we will remove access to the work immediately and investigate your claim. 


\title{
$125 \mathrm{GHz}$ microwave signal generation employing an integrated pulse shaper
}

\author{
Shasha Liao, Yunhong Ding, Jianji Dong, ${ }^{*}$ Member, IEEE, Xu Wang, and Xinliang Zhang, Member, \\ IEEE
}

\begin{abstract}
We propose and experimentally demonstrate an on-chip pulse shaper for $125 \mathrm{GHz}$ microwave waveform generation. The pulse shaper is implemented based on a silicon-on-insulator (SOI) platform that has a structure with eight-tap finite impulse response (FIR). And there is an amplitude modulator on each tap. By controlling the thermal heaters on the amplitude modulators, we obtain several signals centered at 125 GHz with typical envelopes, such as square envelope, triangular envelope, sawtooth envelope, Gaussian envelope and so on. Our scheme has some significant advantages, such as the central frequency of the generated microwave waveforms is larger than $100 \mathrm{GHz}$, and it has wide bandwidth when changing the time delay of the adjacent taps. And compactness, capability for integration with electronics and small power consumption are also its merits.
\end{abstract}

Index Terms - Microwave integrated circuit, Signal generators, Pulse shaping method.

\section{INTRODUCTION}

$\mathrm{H}$ igh frequency microwave signal generation has significant applications in several different fields such as electronic countermeasure, radar system [1], wireless communications [2], sensing [3] and so on [4, 5]. The high frequency microwave system has some advantages over the traditional microwave system in which the frequency of the microwave is from 1 to 40 GHz. For example, the high frequency microwave communication has much broader bandwidth and it is not so sensitive to climate change. And the high frequency microwave radar has narrow beams which can improve the resolution of the observation. However, the electronic methods of high frequency microwave signal generation are limited by the bandwidth and tunability. So it is desirable to generate microwave signal by photonic methods [6, 7]. In 1990s and 2000s numerous schemes of single frequency microwave signal generation have been proposed which are based on different techniques including optical injection locking [8], phased-lock loop [9], and dual-wavelength laser source [10]. However, the

Shasha Liao, Jianji Dong, Xu Wang and Xinliang Zhang are with Wuhan National Laboratory for Optoelectronics, Huazhong University of Science and Technology, Wuhan 430074, China. (e-mail: zgcqlss24@163.com; jjdong@mail.hust.edu.cn; lianxue921125qing@qq.com; xlzhang@mail.hust. edu.cn ).

Yunhong Ding is with Department of Photonics Engineering, Technical University of Denmark, 2800 Kgs. Lyngby, Denmark (e-mail: yudin@fotonik,dtu.dk ). optical schemes of microwave waveform generation attract more and more attentions nowadays. There are several different kinds of schemes have been proposed. The scheme based on space-to-time mapping is able to tune precise spectra and this method is widely used in optical arbitrary waveform generation [11]. But it requires bulky diffractive optical element and it is sensitive to the environmental fluctuation and low power efficiency. Another alternative scheme is using frequency-to-time mapping $[12,13]$. The schemes proposed by Andrew M. Weiner et al and J. Yao et al have generated microwave waveforms with good performance and they also generated chirped microwave pulses. While microwave waveforms over $100 \mathrm{GHz}$ are very difficult to obtain because the restraint of mapping. Furthermore, the on-chip mapping device with adequate large dispersion is still difficult to achieve. So these schemes are all discrete schemes. There are also other methods for microwave waveform generation, such as using Fourier synthesis [1, 14] and finite impulse response (FIR) filter $[15,16]$. Some of them are integrated schemes [14, 17] with the common advantages of compactness and low power consumption. But the schemes based on Fourier synthesis method require spectral dispersers with high resolution which will reduce the fabrication tolerance, and make large scale fabrication difficult. And the maximum central frequency of the generated microwave in these schemes is only $80 \mathrm{GHz}$ [17].

In the paper, we propose and demonstrate a photonic high frequency microwave signal generator based on an eight-tap FIR silicon integrated circuit. By adjusting the amplitude of each tap by thermal heaters, we obtain several microwave signals centered at $125 \mathrm{GHz}$ with typical envelopes, such as square envelope, triangular envelope, sawtooth envelope and Gaussian envelope, etc. Comparing to previous schemes, the central frequency of the generated microwave signal is 125 $\mathrm{GHz}$ which is much higher than $80 \mathrm{GHz}$ (previous record), and it is integrated on a silicon on insulator (SOI) wafer so it has the capability for integration with electronics and low power consumption is also its merits. Furthermore, our shaper does not require any dispersers, therefore, it has large fabrication tolerance and is prone to large scale integration. And the microwave frequency is decided by the time delay between the adjacent taps, the central frequency can vary from $33.3 \mathrm{GHz}$ to $200 \mathrm{GHz}$ by the same structure, if only, the time delay changes from 5 ps to 30 ps $[18,19]$, which is a very wide bandwidth. 


\section{PRINCIPLE}

The pulse shaper is based on an eight-tap FIR structure. The schematic diagram is shown in Fig. 1 and it is integrated on an SOI wafer. This is how our pulse shaper would work: Firstly, the cascaded multimode interferometer (MMI) couplers divide the input signal into eight taps. Secondly, the light propagates through a series of time delays which are realized by different lengths of waveguide, and we can assume that the time delay between two adjacent taps is $\tau$. And then, the light on each tap is amplitude modulated by amplitude modulator which is realized by Mach-Zehnder interferometer (MZI) with a heater on one of its arms. Assuming that the amplitudes of the eight taps are $\alpha_{1}$, $\alpha_{2}, \alpha_{3}, \alpha_{4}, \alpha_{5}, \alpha_{6}, \alpha_{7}, \alpha_{8}$, respectively. The temporal response of the pulse shaper can be expressed as

$$
h(t)=\sum_{i=1}^{8} \alpha_{i} h_{0}(t) * \delta(t-i \tau)
$$

where $h_{0}(t)$ is the input pulse, $\delta(t)$ is unit impulse function. Equation (1) indicates that the interval of the pulse burst is determined by the time delay $\tau$, and the central microwave frequency of the output signal is reciprocal of the interval. And the generated waveforms can be reshaped by changing the amplitude modulation factors of the eight taps. In the scheme, we use wire-bonding technique to contact the metal electrode pads on chip with the external wire.

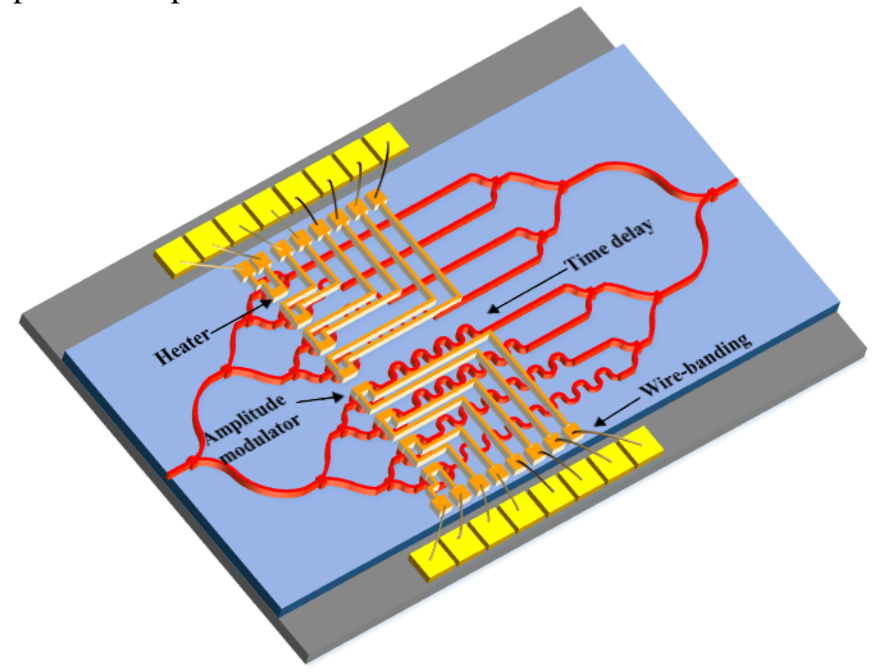

Fig. 1. Schematic diagram of the proposed on-chip pulse shaper

\section{EXPERIMENT}

Figure 2 shows the microscopic image of the fabricated pulse shaper. The SOI wafer we used has a $250 \mathrm{~nm}$ thick top silicon layer and $3 \mu \mathrm{m}$ thick buried oxide (BOX). And the waveguide is fully etched, so the height of the waveguide is also $250 \mathrm{~nm}$. The grating couplers we used in our pulse shaper are the fully etched apodized grating couplers [20]. The fabrication process is similar to that reported in our previous paper [21]. Firstly, we fabricate the waveguide and the gratings. Secondly, a $700 \mathrm{~nm}$ thick silica is deposited on the sample. Then, anther $700 \mathrm{~nm}$ thick boro-phospho-silicate-glass (BPSG) layer is deposited in order to planarize the surface. At last, $100 \mathrm{~nm}$ Ti heaters are formed followed by metal deposition and lift-off after the top glass layer is thinned to $1 \mu \mathrm{m}$. And we use thick gold electrodes for wire-bonding in this chip. The loss of the grating couplers is $3 \mathrm{~dB} /$ port, and the loss of the waveguide is $2 \mathrm{~dB} / \mathrm{cm}$. The fiber-to-fiber loss of our pulse shaper is about $17 \mathrm{~dB}$ when there is no electricity supplied onto the pulse shaper. The length of the heaters is about $180 \mu \mathrm{m}$. And the rise time and fall time of the thermal-optic modulators are about $66 \mu \mathrm{s}$ and $27 \mu \mathrm{s}$, respectively [22]. The relative time delay of the eight taps is 8 ps, so the central frequency of the microwave signals is 125 $\mathrm{GHz}$ by our pulse shaper. And the size of our pulse shaper is about $5 \mathrm{~mm}^{2}$.

The experimental setup we used is shown in Fig. 3 to generate several typical microwave signals, which is used to test the performance of our pulse shaper. An ultra-short pulse is emitted from a mode-lock laser (MLL) with $10 \mathrm{GHz}$ repetition frequency. And two vertical grating couplers are used to couple the light from fiber to silicon waveguide and the reverse direction. A polarization controller (PC) is placed before the input grating coupler because the grating couplers are sensitive to the polarization. The electrode pads of the amplitude array on chip are bonded with the electrodes on printed circuit board (PCB) and the external wires are pasted on the electrodes by conductive adhesive. We use independent power supplies to generate variable voltages which are applied to different modulators by the external wire. The wire-bonding technique greatly simplifies the experiment operations. A band-pass filter with $2.5 \mathrm{~nm}$ band width is used to reduce the noise effect. And at last the output temporal waveform is measured by an oscilloscope (OSC) with a bandwidth of $500 \mathrm{GHz}$ (Eye-Checker 1000C).

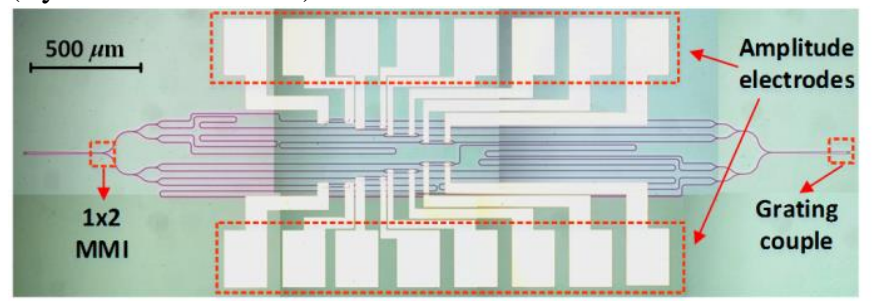

Fig. 2. Metallurgical microscopy image of the on-chip pulse shaper

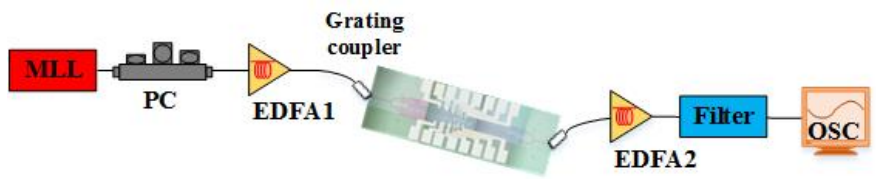

Fig. 3. Experimental setup of the high frequency microwave signal generation with employing the on-chip pulse shaper. (MLL: mode-lock laser, PC: polarization controller, EDFA: erbium-doped fiber amplifier, OSC: oscilloscope.)

Figure 4(a) shows the input pulse launched into the pulse shaper (including the band-pass filter), the full width at half maximum (FWHM) of the input pulse is $3.4 \mathrm{ps}$ and the signal to noise ratio (SNR) is $61 \mathrm{~dB}$. The SNR is calculated from the spectrum of the measured waveform. The repetition rate of the input pulse is $10 \mathrm{GHz}$. An example of the measured signal with square envelope is shown in Fig. 4(b). The ideal amplitude array of this case should be $\alpha=[1,1,1,1,1,1,0,0]$, the actual amplitude array is $\alpha=[1,0.87,0.87,0.90,0.86,0.94,0,0]$. Because of the unbalance power of each tap, the power of the seventh and the eighth taps are much smaller than other taps. So we set two " 0 "s at the seventh and the eighth taps. The FHWM 
of the measured waveform is $43.45 \mathrm{ps}$, and the SNR of the output waveform is $59.3 \mathrm{~dB}$. The time interval between the adjacent peaks is about $8.1 \mathrm{ps}$ and the output waveform is shown as blue solid line. In order to obtain this waveform, we should control the amplitude modulators by adjusting all the voltages applied on the electrodes so that each peak matches well with the envelope of the ideal microwave waveform. With similar method, the signal with isosceles triangular envelope can also be achieved, which is shown in Fig. 4(c) as the blue solid line. The actual amplitude array is $\alpha=[0.26,0.44,0.65,1$, $0.67,0.51,0.24,0]$, and the FHWM of the measured waveform is $25 \mathrm{ps}$ and SNR is $62.1 \mathrm{~dB}$. Figure 4(d) shows a signal with trapezoid envelope, and the actual amplitude array is $\alpha=[1,0.84$, $0.77,0.78,0.79,0.58,0.45,0]$. The FHWM of the measured waveform is $42.91 \mathrm{ps}$ and SNR is $61.8 \mathrm{~dB}$. Two opposite signals with sawtooth envelopes are shown in Figs. 4(e) and 4(f) with the FWHMs of $26.6 \mathrm{ps}$ and $19.0 \mathrm{ps}$, respectively. The SNRs of the two waveforms are $62.3 \mathrm{~dB}$ and $56.3 \mathrm{~dB}$, respectively. The amplitude arrays are $\alpha=[1,0.82,0.71,0.65$, $0.45,0.32,0.21,0]$ and $\alpha=[0.11,0.28,0.53,0.74,0.85,1,0,0]$, respectively. Figures $4(\mathrm{~g})$ and $4(\mathrm{~h})$ are two opposite signals with oblique triangular envelopes, and the FWHMs of them are $18.9 \mathrm{ps}$ and $18.61 \mathrm{ps}$, respectively. The SNRs are $61.6 \mathrm{~dB}$ and $63.1 \mathrm{~dB}$, respectively. The amplitude arrays are $\alpha=[0.32,0.64,1$, $0.82,0.46,0.38,0.16,0]$ and $\alpha=[0.08,0.28,0.49,0.67,1,0.83$, $0.32,0]$, respectively. Furthermore, we obtain a pulse burst with Gaussian envelop and with the FWHM of 33.9 ps, which is shown in Fig. 4(i), and the SNR of the waveform is $59.6 \mathrm{~dB}$. The actual amplitude array is $\alpha=[0.45,0.67,0.83,1,0.95,0.74$, $0.48,0]$. And Figure 4(j) shows a pulse burst with super-Gaussian envelope, and the actual amplitude array is $\alpha=[0.49,0.81,0.95,1,0.99,0.89,0.53,0]$. The FWHM and SNR of this waveform are $35.2 \mathrm{ps}$ and $62.3 \mathrm{~dB}$. To comparing the generated waveforms with the ideal ones, the ideal waveforms are also shown as the red dash line. As shown in Fig. 4 , the measured waveforms all fit well with the ideal ones.

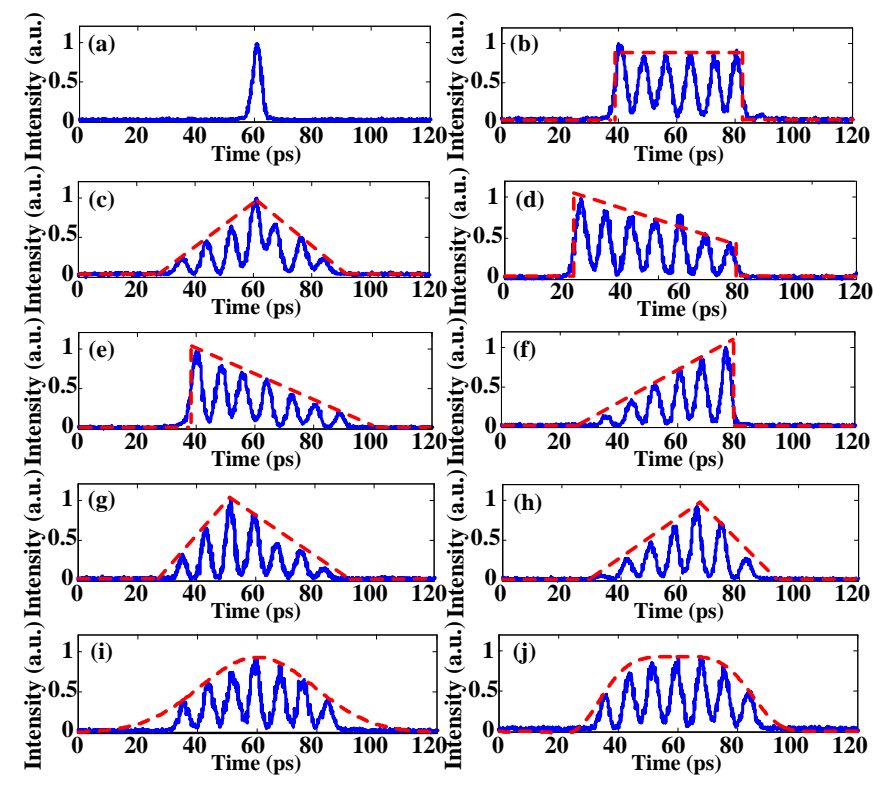

Fig. 4. (a) Input pulse, measured waveforms (blue solid line) and ideal microwave waveforms (red dash line) of (b) $125 \mathrm{GHz}$ pulse burst with square envelope (the amplitude array is $[1,0.87,0.87,0.90,0.86,0.94,0,0]$ ), (c) 125 $\mathrm{GHz}$ pulse burst with isosceles triangular envelope (the amplitude array is $[0.26$, $0.44,0.65,1,0.67,0.51,0.24,0]$ ), (d) $125 \mathrm{GHz}$ pulse burst with trapezoid envelope (the amplitude array is $[1,0.84,0.77,0.78,0.79,0.58,0.45,0])$, (e) and (f) $125 \mathrm{GHz}$ pulse bursts with sawtooth envelope (the amplitude arrays are $[1,0.82,0.71,0.65,0.45,0.32,0.21,0]$ and $[0.11,0.28,0.53,0.74,0.85,1,0,0]$, respectively), (g) and (h) $125 \mathrm{GHz}$ pulse bursts with oblique triangular envelope (the amplitude array are $[0.32,0.64,1,0.82,0.46,0.38,0.16,0]$ and $[0.08,0.28,0.49,0.67,1,0.83,0.32,0]$, respectively), (i) $125 \mathrm{GHz}$ pulse burst with Gaussian envelope (the amplitude array is $[0.45,0.67,0.83,1,0.95,0.74$, $0.48,0]$ ) and (j) $125 \mathrm{GHz}$ pulse burst with super-Gaussian envelope (the amplitude array is $[0.49,0.81,0.95,1,0.99,0.89,0.53,0])$.

And we also calculate the cross-correlation coefficients between the measured waveforms and the pulse bursts with ideal envelops, the cross-correlation coefficient can be expressed as

$$
C_{c}=\frac{\int_{T} h_{m}(t) \cdot h_{i}(t) d t}{\sqrt{\int_{T} h_{m}^{2}(t) d t \cdot \int_{T} h_{i}^{2}(t) d t}}
$$

where $h_{m}(t)$ is the measured waveform and $h_{i}(t)$ is the pulse burst with ideal envelop, and the result is shown in Table 1. All cross-correlation coefficients are larger than $97 \%$, that is to say, our scheme has a good performance on waveform generation. Table 1. Cross-correlation coefficient between the measured waveforms and the pulse bursts with ideal envelops.

\begin{tabular}{|c|c|c|c|}
\hline Type & Square & Isosceles triangular & Trapezoid \\
\hline$C_{c}$ & $99.14 \%$ & $98.08 \%$ & $98.76 \%$ \\
\hline Type & Sawtooth1 & Sawtooth2 & Oblique triangular1 \\
\hline$C_{c}$ & $98.92 \%$ & $99.29 \%$ & $98.5 \%$ \\
\hline Type & Oblique triangular2 & Gaussian & Super-Gaussian \\
\hline$C_{c}$ & $97.92 \%$ & $97.82 \%$ & $98.29 \%$ \\
\hline
\end{tabular}

In order to test the performance of our microwave signals, we calculate the microwave spectra via Fourier transform of output signals, which are shown in Fig. 5. Figure 5(a1) shows the calculated power of the microwave spectrum of the signal with square envelope as the blue solid line. And it is normalized. Because the output signal is periodic and the time period is 100 $\mathrm{ps}$, the microwave spectrum is a group of comb lines with 10 $\mathrm{GHz}$ spacing. The red dash line shown in Fig. 5(a1) is the envelope of the spectrum, which can be calculated by picking out one period of the signal and then processing by Fourier transform. The mainlobe-sidelobe suppression ratio (MSSR) is 11.4dB. Figure 5(a2) shows the calculated phase of the microwave spectrum of the signal with square envelope. Figures 5(b1) to 5(f2) are the calculated microwave spectra of signal with isosceles triangular envelope (5(b1) and 5(b2)), signal with trapezoid envelope (5(c1) and 5(c2)), sawtooth microwave waveform $(5(\mathrm{~d} 1)$ and $5(\mathrm{~d} 2)$, its waveform is shown in Fig. 4(e)), signal with Gaussian envelope (5(e1) and 5(e2)), and signal with super-Gaussian envelope (5(f1) and 5(f2)). The MSSRs are $10.23 \mathrm{~dB}, 11.48 \mathrm{~dB}, 10.4 \mathrm{~dB}, 16.5 \mathrm{~dB}$ and $15.7 \mathrm{~dB}$, respectively. The central frequencies of the mainlobe are all $125 \mathrm{GHz}$ in Fig. 5. 

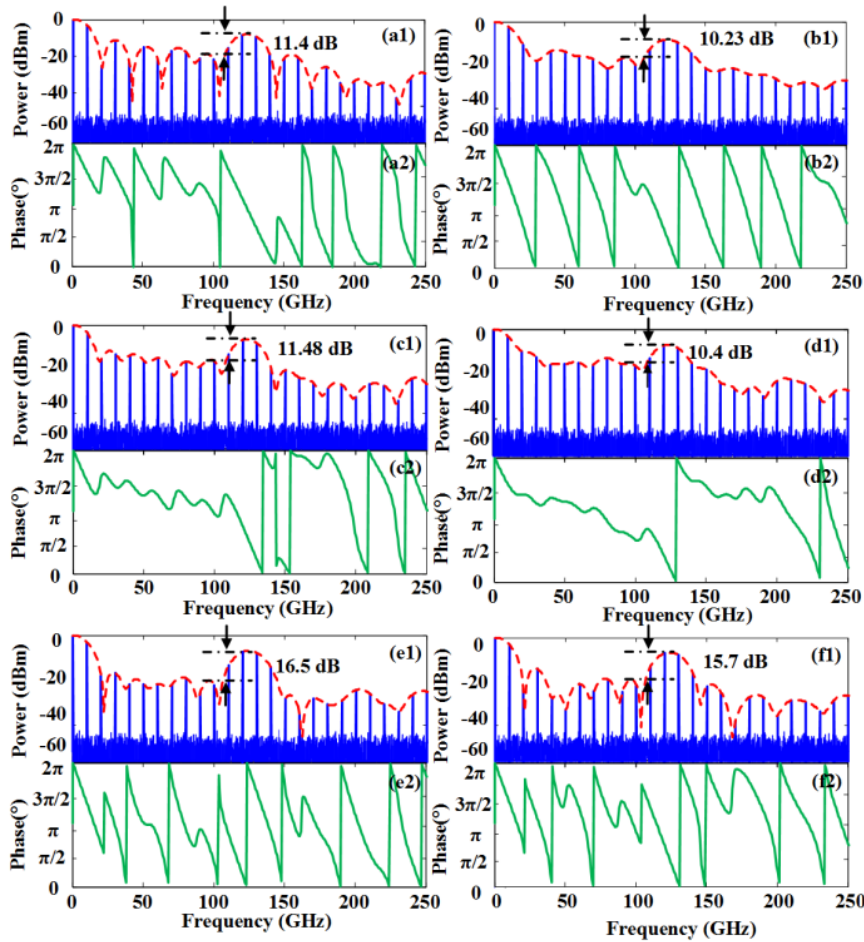

Fig. 5. Calculated microwave spectra (power: blue solid line and phase: green solid line) and envelope of the spectra (red dash line) via Fourier transform of output signals of (a1) and (a2) signal with square envelope where the MSSR is $11.4 \mathrm{~dB}$, (b1) and (b2) signal with isosceles triangular envelope where the MSSR is $10.23 \mathrm{~dB},(\mathrm{c} 1)$ and (c2) signal with trapezoid square envelope where the MSSR is $11.48 \mathrm{~dB},(\mathrm{~d} 1)$ and (d2) signal with sawtooth envelope where the MSSR is $10.4 \mathrm{~dB}$, (e1) and (e2) signal with Gaussian envelope where the MSSR is $16.5 \mathrm{~dB}$, (f1) and (f2) signal with super-Gaussian envelope where the MSSR is $15.7 \mathrm{~dB}$.

The key component of the pulse shaper is the amplitude modulators, so complete amplitude control is very important in our scheme. Figure 6(a) shows the output power varying with the voltage applied on to the electrode as the blue dots. The red solid line is a numerical fitting of the measured dot. And Figure 6(b) shows three examples of different output power of the first tap, and the voltages applied on to the electrodes are also shown in the figure. We can see that the amplitude control is complete in our pulse shaper from Fig. 6. And our pulse shaper has good stability and low crosstalk among the heaters. When we achieve different waveforms, the power consumption changes. And we have recorded all voltages and electric currents of all heaters for different waveforms to calculate power consumptions, and the maximum one is about $210 \mathrm{~mW}$.
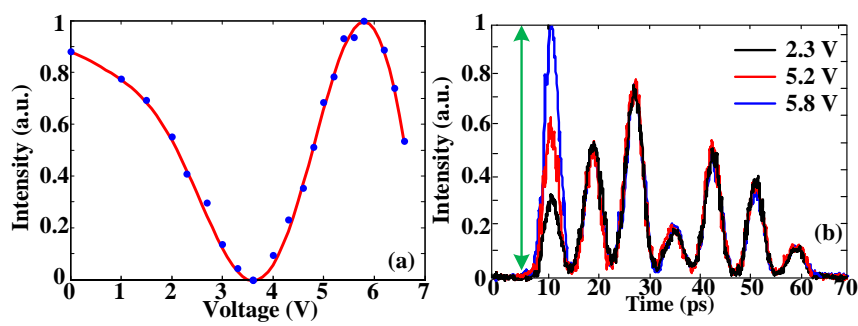

Fig. 6. (a) Output power varies with the voltage applied on to the electrode (measured power is shown as blue dot and numerical fitting is shown as red solid line), and (b) three examples of the different output power of the first tap.

In our pulse shaper, the delay line is realized by different length of waveguide, so the time delay is fixed. We can add a group of chirped waveguide gratings [23] to obtain tunable time delays. Thus we can implement a tunable high frequency microwave signal generator and the layout of the generator is shown in Fig. 7.

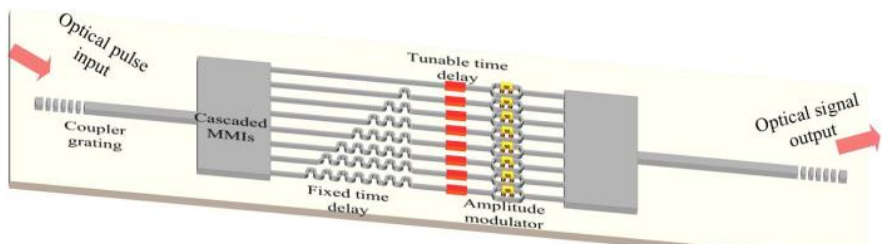

Fig. 7. Layout of the full integration of a tunable high frequency microwave signal generator.

\section{CONCLUSION}

We have proposed and demonstrated a photonic high frequency microwave signal generator centered at $125 \mathrm{GHz}$ based on an FIR silicon integrated circuit. By controlling the amplitude modulator of each tap, we have generated several typical waveforms such as pulse bursts with square envelope, with isosceles triangular envelope, with trapezoid envelope, with sawtooth envelope, with oblique triangular envelope, with Gaussian envelope and with super-Gaussian envelope. In order to test the performance of our microwave signals, we also calculate the microwave spectra via Fourier transform of output signals. The MSSRs of the generated signals are all larger than $10 \mathrm{~dB}$. Furthermore, we verify the complete amplitude control of our pulse shaper. Our scheme has some significant advantages, such as it has wide bandwidth and it is compactness. And capability for integration with electronics and small power consumption are also its merits.

\section{ACKNOWLEDGMENT}

The research is supported under New Century Excellent Talents in Ministry of Education of China (NCET-11-0168), National Excellent Doctoral Dissertation of China (201139), and National Natural Science Foundation of China (61622502, 11374008, and 61475052)

\section{REFERENCES}

[1] Jiang, Y., Ma, C., Bai, G., Qi, X., Tang, Y., Jia, Z., Zi, Y., Huang, F., and $\mathrm{Wu}, \mathrm{T}$., "Photonic microwave waveforms generation based on time-domain processing," Opt Express vol. 23, pp. 19442-19452 2015.

[2] McKinney, J. D., Lin, I. S., and Weiner, A. M., "Shaping the power spectrum of ultra-wideband radio-frequency signals," Ieee T Microw Theory vol. 54, pp. 4247-4255 2006.

[3] Dumeige, Y., and Féron, P., "Coupled optical microresonators for microwave all-optical generation and processing," Opt Lett vol. 40, pp. 3237-3240 2015.

[4] Latkin, A. I., Boscolo, S., Bhamber, R. S., and Turitsyn, S. K., "Doubling of optical signals using triangular pulses," JOSA $B$ vol. 26, pp. 1492-1496 2009.

[5] Yao, J., "Photonic generation of microwave arbitrary waveforms," Opt Commun vol. 284, pp. 3723-3736 2011.

[6] Capmany, J., Li, G., Lim, C., and Yao, J., "Microwave photonics: current challenges towards widespread application," Opt Express vol. 21, pp. 22862-22867 2013.

[7] Li, W., and Yao, J., "Microwave generation based on optical domain microwave frequency octupling," Ieee Photonic Technol L vol. 22, pp. 24-26 2010. 
[8] Goldberg, L., Taylor, H., Weller, J., and Bloom, D., "Microwave signal generation with injection-locked laser diodes," Electronics Letters vol. 19, pp. 491-493 1983

[9] Ramos, R., and Seeds, A., "Fast heterodyne optical phase-lock loop using double quantum well laser diodes," Electronics letters vol. 28, pp. 82-83 1992.

[10] Chen, X., Deng, Z., and Yao, J., "Photonic generation of microwave signal using a dual-wavelength single-longitudinal-mode fiber ring laser," Ieee T Microw Theory vol. 54, pp. 804-809 2006.

[11] McKinney, J., Leaird, D., and Weiner, A., "Millimeter-wave arbitrary waveform generation with a direct space-to-time pulse shaper," Opt Lett vol. 27, pp. 1345-1347 2002.

[12] Dezfooliyan, A., and Weiner, A. M., "Photonic synthesis of high fidelity microwave arbitrary waveforms using near field frequency to time mapping," Opt Express vol. 21, pp. 22974-22987 2013.

[13] Wang, C., and Yao, J., "Photonic generation of chirped millimeter-wave pulses based on nonlinear frequency-to-time mapping in a nonlinearly chirped fiber Bragg grating," Microwave Theory and Techniques, IEEE Transactions on vol. 56, pp. 542-553 2008

[14] Wang, J., Shen, H., Fan, L., Wu, R., Niu, B., Varghese, L. T., Xuan, Y., Leaird, D. E., Wang, X., and Gan, F., "Reconfigurable radio-frequency arbitrary waveforms synthesized in a silicon photonic chip," Nature communications vol. 62015.

[15] Samadi, P., Chen, L. R., Callender, C., Dumais, P., Jacob, S., and Celo, D., "RF arbitrary waveform generation using tunable planar lightwave circuits," Opt Commun vol. 284, pp. 3737-3741 2011.

[16] Adams, R., Ashrafi, R., Wang, J., Dizaji, M. R., and Chen, L. R., "RF-arbitrary waveform generation based on microwave photonic filtering," Photonics J, IEEE vol. 6, pp. 1-8 2014.

[17] Samadi, P., Chen, L., Callender, C., Dumais, P., Jacob, S., and Celo, D., "RF arbitrary waveform generation using tunable planar lightwave circuits," Opt Commun vol. 284, pp. 3737-3741 2011.

[18] Wang, X., Liao, S., and Dong, J., "Optical true time delay based on contradirectional couplers with single sidewall-modulated Bragg gratings," (2016), pp. 100260C-100260C-100266.

[19] Tan, D. T. H., Ikeda, K., Saperstein, R. E., Slutsky, B., and Fainman, Y., "Chip-scale dispersion engineering using chirped vertical gratings," Opt Lett vol. 33, pp. 3013-3015 2008.

[20] Ding, Y., Ou, H., and Peucheret, C., "Ultrahigh-efficiency apodized grating coupler using fully etched photonic crystals," Opt Lett vol. 38, pp. 2732-2734 2013.

[21] Liao, S., Ding, Y., Dong, J., Yang, T., Chen, X., Gao, D., and Zhang, X., "Arbitrary waveform generator and differentiator employing an integrated optical pulse shaper," Opt Express vol. 23, pp. 12161-12173 2015.

[22] Liao, S., Ding, Y., Dong, J., Yan, S., Wang, X., and Zhang, X., "Photonic arbitrary waveform generator based on Taylor synthesis method," Opt Express vol. 24, pp. 24390-24400 2016.

[23] St-Yves, J., Bahrami, H., Jean, P., LaRochelle, S., and Shi, W., "Widely bandwidth-tunable silicon filter with an unlimited free-spectral range," Opt Lett vol. 40, pp. 5471-5474 2015.
Shasha Liao received the B.S. degree in optoelectronics science and technology from the Huazhong University of Science and Technology, Wuhan, China, in 2012, where she is currently working toward the Ph.D. degree in Wuhan National Laboratory for Optoelectronics.

She is currently working on arbitrary waveform generation.

Jianji Dong received the Ph.D. degree in optoelectronics engineering from the Huazhong University of Science and Technology (HUST), Wuhan, China, in 2008.

From Feb. 2006 to Aug. 2006, he worked in Network Technology Research Centre, Nanyang Technological University, Singapore, as an Exchange Student. From Nov. 2008 to Feb. 2010, he worked in the Centre of Advanced Photonics and Electronics, Cambridge University, U.K., as a Research Associate. He is currently a full Professor in the Wuhan National Laboratory for Optoelectronics, HUST. He is working on silicon photonics devices, and integrated microwave photonics. He has made some contributions in broad areas, such as ultrafast photonic differentiator using silicon waveguides, microwave signal processing, ultra-wide band signal generation and modulation, and so forth. He has published more than 80 journal papers, including OSA, IEEE group. He is an Editorial Board Member of "Scientific Reports". He received the National Best Dissertations Award in 2010 and the first award of Natural Science of Hubei Province in 2013.

Xinliang Zhang received the Ph.D. degree from the Huazhong University of Science and Technology (HUST), Wuhan, China, in 2001.

$\mathrm{He}$ is currently a Professor with the Wuhan National Laboratory for Optoelectronics and the Dean of School of Optical and Electronic Information, HUST. He has published 200 related papers in international journals or conferences proceedings. His current research interests include all-optical signal processing and silicon photonics. 